\title{
Pickup of Essential Kinematics Underpins Expert Perception of Movement Patterns
}

\author{
Bruce Abernethy \\ Institute of Human Performance \\ The University of Hong Kong \\ and School of Human Movement Studies \\ The University of Queensland, Brisbane, Australia
}

\author{
Khairi Zawi \\ Department of Physical Education \\ University Putra Malaysia, Selangor \\ and School of Human Movement Studies, \\ The University of Queensland, Brisbane, Australia
}

\begin{abstract}
In a series of 3 experiments, the authors examined the ability of badminton players of different skill levels (12 experts and 12 nonexperts) to anticipate the direction of badminton strokes. Participants viewed either film or point-light displays under a range of temporal or spatial occlusion conditions. Worldclass players were able to consistently pick up useful predictive information from the advance (precontact) kinematics of both the lower body and the racquet when the motion of those features was presented in isolation, whereas recreational players' use of the same information depended on the concurrent presence of linked segments. Participants' information pickup closely matched key biomechanical changes in the movement pattern being viewed, although, contrary to a common-coding view of perception and action (e.g., W. Prinz, 1997), some important differences were evident between the characteristics of the experts' movement prediction and those of expert movement production.
\end{abstract}

Keywords: expertise, kinematics, perception, skill learning

E arly and accurate anticipation of future events is a defining characteristic of expert performance in many everyday work and sport tasks. However, limited understanding of what perceptual information provides the basis for skilled anticipation constrains the design of practice methods that can systematically enhance anticipatory skill. In this article, we describe an approach to determining the informational underpinnings of skilled perception and anticipation in a sport setting. We based our approach on a comparison of domain-specific experts' and less skilled participants' prediction performances. We used the specific task of anticipating an opponent's stroke in the racquet sport of badminton to examine expertise-related differences in information use. The time constraints under which the receiving player must operate in badminton make the early perception of the opponent's intention, and the avoidance of deception, fundamental to success in that task.

In studies of expert perception in racquet sports, researchers have generally used variable levels of temporal occlusion of vision of an opponent's hitting action to determine skillrelated differences in advance information pickup. Early temporal occlusion studies (e.g., Enberg, 1968) consistently revealed superior task performance by expert players under conditions in which occlusion occurred at or before the point of racquet-ball contact. That finding offers researchers an explanation of how skilled performers in natural tasks can commence their movement responses earlier, alleviate the usual processing constraints that reaction time and movement time impose (cf. Salthouse, 1991), and provide the impression of having "all the time in the world" (Bartlett, 1947).

In later racquet sport studies, investigators were able to more precisely describe the time course of advance pickup of information from the opponent's movement pattern by using multiple precontact occlusion times. For example, Abernethy and Russell (1987) had expert and novice badminton players predict the landing position of badminton strokes viewed under five different levels of temporal occlusion. In the most difficult condition (t1), they permitted players to view the opponent's hitting action only until $167 \mathrm{~ms}$ before racquet-shuttle contact. In the other conditions, they occluded the display $83 \mathrm{~ms}$ before contact (t2), at the point of contact (t3), 83 ms after contact (t4), and at a time following the last outward flight of the struck shuttle (t5). Each occlusion point provided about $83 \mathrm{~ms}$ of

Correspondence address: Bruce Abernethy, Institute of Human Performance, The University of Hong Kong, FHSC, 111-113 Pokfulam Road, Hong Kong. E-mail address: bruceab@hkucc.hku.hk 
additional information; therefore, comparison of prediction accuracy across adjacent occlusion conditions provided a measure of information pickup within the time window bounded by the adjacent occlusion conditions. When Abernethy and Russell used that approach, they found that experts were able to pick up significant information over three time windows ( $\mathrm{t} 1-\mathrm{t} 2, \mathrm{t} 2-\mathrm{t} 3$, and $\mathrm{t} 3-\mathrm{t} 4)$, whereas novices could significantly reduce their error in predicting stroke-landing position only over two such periods ( $\mathrm{t} 2-\mathrm{t} 3$ and $\mathrm{t} 3-\mathrm{t} 4)$. Although novices were able to pick up advance information from the opponent's precontact movement pattern (in the $\mathrm{t} 2-\mathrm{t} 3$ window), they picked up their first significant information later in the movement sequence than did the experts. Experts were picking up information at least as early as the period 167-83 ms before contact. Because the arm holding the racquet underwent its greatest displacement during the $\mathrm{t} 1-\mathrm{t} 2$ period, whereas the racquet underwent its greatest displacement in the $\mathrm{t} 2 \mathrm{-} \mathrm{t} 3$ period, Abernethy and Russell gleaned some support for the notion that experts are attuned not only to earlier information on the opponent's action but also to information from spatially distinct regions. Improvements from $\mathrm{t} 3$ to $\mathrm{t} 4$, which were evident for both skill groups, were presumably a function of the availability of shuttle flight information.

To examine more systematically possible expert-novice differences in the spatial location of anticipatory information, Abernethy and Russell (1987) also used a spatial occlusion procedure. In that procedure, they temporally occluded all displays at the point of racquet-shuttle contact and they masked visibility to selected spatial areas of the opponent's movement pattern throughout the hitting action. Masking of visibility of (a) the racquet and (b) the racquet plus the arm holding it caused significant decrements in prediction accuracy for all participants, although the effect was a differential one. Experts' prediction of stroke landing position was significantly poorer when Abernethy and Russell occluded the arm in addition to the racquet, whereas the novices' was not, suggesting, in concordance with the temporal occlusion findings, that experts were able to pick up useful anticipatory information from not only the motion of the racquet but also the motion of the arm holding the racquet. In contrast, novices could pick up advance information only from the racquet. Masking visibility to the lower body and to the head did not disrupt prediction performance for either skill group, indicating either that those regions conveyed no reliable advance information or that whatever information those regions did convey was redundant with the information available before contact from other areas, such as the racquet.

The experts' unique pickup of arm information-information from earlier occurring, more proximal aspects of the hitting action than that of the racquet that novices appeared to rely on-suggests that there may be a strong link between perceptual expertise and the kinematics of the action being viewed. Evidence demonstrating preservation of expert-novice differences in overall anticipatory perfor- mance in tennis (Ward, Williams, \& Bennett, 2002) and in the specific time course of information pickup in the sport of squash (Abernethy, Gill, Parks, \& Packer, 2001) when players view simply the motion of light points displaying information about movement kinematics alone, rather than film displays, supports that proposition. That evidence is not unequivocal, however, and Shim, Carlton, Chow, and Chae (2005) showed in a recent study that expert-novice differences in anticipatory performance that were evident with live and video displays were not present in point-light displays. The existing observations require replication, and more analytical methods are needed to enable researchers to go beyond a description of the nature of expert-novice differences in anticipatory skill to more precisely isolate the specific visual information that experts and novices use. Such information is needed by researchers so that the study of perceptual expertise in sport tasks can usefully contribute to either the practical development of a principled approach to the provision of practice and instruction or to the conceptual development of a more generic understanding of perceptual expertise as it occurs in natural tasks with significant action components.

Over the past decade, Prinz and coworkers (e.g., Hecht, Vogt \& Prinz, 2001; Hommel, Musseler, Aschersleben, \& Prinz, 2001; Prinz, 1997) have contributed significantly to the development of conceptual understanding of the overall relationship between perception and action through their formulation of the common-coding hypothesis. According to that hypothesis, the perception and the production of the same action involve common coding so that the task of perceiving and anticipating someone else's movement patterns triggers the perceivers' coded representations for their own production of the same action. Both (a) neural evidence of the presence in the human brain of so-called mirror neurons that discharge when a particular action is either produced or observed (e.g., Buccino, Binkofski, \& Riggio, 2004; Buccino \& Riggio, 2006) and (b) recent functional magnetic resonance imaging (fMRI) evidence showing that the mirror system's response depends on whether the observed skill has been personally acquired have supported that hypothesis. Calvo-Merino, Glaser, Grèzes, Passingham, and Haggard (2005), for example, showed greater brain activity in the mirror system of expert ballet dancers viewing video clips of different dance routines than in the brains of unskilled dancers watching the same clips. Collectively, those two lines of evidence suggest that humans' perceptual expertise may arise because their brains simulate the production of the action being viewed.

Because experts in sports tasks such as badminton are superior to nonexperts not only in perception but also in stroke production (e.g., Sakurai \& Ohtsuki, 2000), it is plausible that a common-coding mechanism explains their advantage on anticipation tasks of the type used by Abernethy and Russell (1987). Furthermore, because production of expert movement patterns in hitting a badminton stroke involves a characteristic proximal-to-distal summation of 
joint forces and a concomitant proximal-to-distal unfolding of the movement kinematics (e.g., Gowitzke \& Waddell, 1979), and because a greater contribution of proximal segments and a greater exploitation of intersegmental transfer of forces characterize skilled movement production (e.g., Gray, Watts, Debicki, \& Hore, 2006; Kreighbaum \& Barthels, 1996), one might also predict on the basis of a common-coding hypothesis that heightened sensitivity to kinematic changes will be a hallmark of expert perception and anticipation of those strokes.

Our purpose in this study was to examine experimentally the proposition that perceptual expertise in natural tasks is linked directly to the pickup of essential display kinematics and, through that examination, also to test some predictions that can be derived from the common-coding hypothesis. We first determined the replicability of existing findings on the temporal and spatial location of informational sources used by badminton players of different skill levels and examined the nature of expert-nonexpert differences in advance information pickup when the only observable information was that arising from the opponent's kinematics. We then undertook a more analytic determination of the key kinematic features within an opponent's action that provide useful advance information about the opponent's intentions and explored the nature of expertise-related differences in attunement to those information sources.

We report on three experiments, in each of which we examined the time course of anticipatory information pickup through the use of multiple temporal occlusion points. In Experiment 1, we compared the abilities of expert and nonexpert badminton players to make predictions on the basis of film displays and point-light displays that provided only kinematic information. In Experiment 2, we examined advance information pickup across point-light displays that presented only the isolated kinematics of specific elements of the opponent's movement pattern. In Experiment 3, we examined comparative information pickup across pointlight displays in which we progressively added proximal joint motions to the motions of more distal segments. For ease of comparability with previous studies, we derived the displays in all three experiments directly from those used by Abernethy and Russell (1987).

\section{EXPERIMENT 1}

Our principal purpose in Experiment 1 was to test the hypothesized linkage between perceptual skill and display kinematics by examining the time course of information pickup in situations in which only kinematic information was available to the players. One can provide solely kinematic information through point-light displays (Johansson, 1973). Although those displays lack the texture, contour, color, and shape cues present in normal film and video displays, they nevertheless provide all the essential information necessary for the correct identification of a host of natural events (e.g., see Brownlow, Dixon, Egbert, \& Radcliffe, 1997; Cutting \& Kozlowski, 1977). Veridical judgments of such things as the identity and gender of an individual from the kinematics of his or her gait patterns from point-light displays are possible, providing the displayed motion does not violate any of the usual biomechanical constraints on human movement (Kourtzi \& Shiffar, 1999). Some evidence supports the possibility that researchers can capture the essential differences between experts and novices in their pickup of advance information in squash and tennis from the kinematics of the opposing player's hitting action (Abernethy et al., 2001; Ward et al., 2002). However, as we noted earlier, researchers do not always observe expertise effects with point-light displays (Shim et al., 2005). In Experiment 1, we examined the specific prediction that an expert advantage would persist equally for point-light displays and for film displays and that the same temporal pattern of information pickup would persist across displays that contain only kinematic information as exists for film displays.

Our subsidiary purpose in Experiment 1 was to determine the extent to which the expert-novice differences in information pickup described by Abernethy and Russell (1987), who used film-based methods, are generalizable to a more elite expert group, a control group with badminton experience but low skill level, and to a dependent measure that is categorical rather than continuous. In keeping with the findings of Abernethy and Russell, we predicted that the expert group would be able to pick up information within the time windows $\mathrm{t} 1-\mathrm{t} 2$ and $\mathrm{t} 2-\mathrm{t} 3$ before contact, whereas the lesser skilled participants would be able to use information only in the latter ( $\mathrm{t} 2-\mathrm{t} 3)$ period.

\section{Participants}

\section{Method}

Participants were 12 expert male badminton players whom we selected from among players and coaches in the Malaysian national training squad plus 12 nonexperts (students from the University Putra Malaysia). All of them participated voluntarily in this experiment as well as in Experiments 2 and 3 . All of the experts either currently held world rankings within the top 100 or had previously held world rankings within the top 20 in the past decade. All the nonexperts had played badminton recreationally but not competitively and had seen international matches played.

\section{Procedure}

All participants completed two tasks - a film occlusion task and a point-light equivalent - that we presented on a large-screen monitor. We counterbalanced the order of presentation of the tasks between participants so that an equal number of participants in each skill group experienced the film task first and the point-light task first. Each task consisted of a series of trials displaying, in either the film or the point-light format, the hitting patterns of a badminton player from the on-court viewing perspective of the player's opponent. On each trial, we required the participants to predict whether the impending direction of the stroke being viewed was cross-court or down-the-line. ${ }^{1}$ They made their 
judgments by circling the appropriate option on a standardized response sheet, and we required them to respond during the 5-s intertrial interval. For each task, we presented a total of 160 trials consisting, in random order, of 32 different badminton strokes, each of which we presented under five different levels of temporal occlusion: $167 \mathrm{~ms}$ before racquet-shuttle contact (t1), $83 \mathrm{~ms}$ before contact (t2), the point of contact (t3), $83 \mathrm{~ms}$ after contact (t4), and after all outward flight of the shuttle that was visible to the camera was completed (t5). Before each task began, we provided 6 practice trials involving strokes that were different from the ones used in the experiment proper.

The film task was the one that Abernethy and Russell (1987, Experiment 1) had used. The point-light task was in all aspects identical to the film task, except that we reduced the display to only 26 disconnected points of light corresponding to the moving position of major landmarks on the player's body (the vertex of the head, the chin-neck intercept, the right and left shoulders, elbow, wrist, middle knuckle, hip, knee, ankle, heel, and toe); the shuttle; and the handle, neck, sides, and head of the racquet. We created the point-light displays by digitizing the original film task frame-by-frame and then representing the spatial location of each point as a white centroid against a black background. Once we had animated the displays, all participants immediately recognized that the point-light displays represented the motion of a badminton player. Each task took approximately $20 \mathrm{~min}$ to complete.

\section{Data Analysis}

We first calculated for each participant the percentage error in predicting stroke direction for each task and then used those percentages in three different analyses. Following an arcsine transformation, we subjected the directional error data to a $2 \times$ $2 \times 5$ (Skill Level $\times$ Display Type $\times$ Occlusion Condition) factorial analyses of variance (ANOVA) with repeated measures on the last two factors. We applied a Greenhouse-Geisser correction to the degrees of freedom in the case of any violations of sphericity, and we computed partial eta-squared $\left(\eta_{\mathrm{p}}{ }^{2}\right)$ values to determine the proportion of total variability attributable to each factor or combination of factors. We sought the sources of any significant effects within the ANOVA model by using the Tukey honestly significant difference procedure. We used the ANOVA to examine the main and interactive effects of skill level, display type, and time of occlusion, with particular interest within each display type in (a) the comparison between the prediction errors of the expert group and the nonexpert group at each of the occlusion conditions and (b) the within-group comparison of prediction error across adjacent temporal occlusion conditions. We determined the 95\% confidence limits for all mean data points for each skill group at each occlusion condition under each display condition to determine if prediction performance was reliably different from the $50 \%$ level that would have arisen simply from guessing. To test for possible carryover benefits from the previous film task exposure, we also conducted a three- way Group $\times$ Condition $\times$ Occlusion ANOVA comparing the prediction performance on the point-light task between those participants who did and those who did not undertake the film task beforehand. We also conducted a comparable analysis of the film task data to determine if previous exposure to the point-light task was in any way facilitatory.

\section{Results}

The omnibus ANOVA revealed significant main effects for skill level, $F(1,22)=60.00, p<.01, \eta_{\mathrm{p}}{ }^{2}=.73$, and occlusion, $F(2.33,51.31)=245.66, p<.01, \eta_{\mathrm{p}}{ }^{2}=.92$. It also revealed significant interactions between the skill and occlusion factors, $F(2.33,51.31)=6.85, p<.01, \eta_{\mathrm{p}}{ }^{2}=.24$, and between the display and occlusion factors, $F(2.05,45.19)=10.59, p<.01$, $\eta_{\mathrm{p}}{ }^{2}=.33$. However, a significant three-way Skill $\times$ Display $\times$ Occlusion interaction, $F(2.05,45.19)=3.38, p<.05, \eta_{\mathrm{p}}{ }^{2}=$ .13 , overrode those effects (see Figure 1).

Post hoc comparisons revealed that experts' error in predicting stroke direction on the film task was significantly lower than that of nonexperts for all temporal occlusion conditions except $\mathrm{t} 1(p<.01)$. Within-group comparisons across time windows revealed significant information pickup (prediction error reduction) in the $\mathrm{t} 2-\mathrm{t} 3$ and $\mathrm{t} 3-\mathrm{t} 4$ time windows for both experts and nonexperts. The changes in prediction error across the other adjacent occlusion conditions were nonsignificant $(p>.05)$. The mean prediction error for the experts was significantly better than chance (guessing) levels in all occlusion conditions, whereas nonexperts' prediction at the $\mathrm{t} 2$ condition was not reliably different from chance. For the point-light task, both skill groups significantly reduced their directional error across the $\mathrm{t} 2-\mathrm{t} 3, \mathrm{t} 3-\mathrm{t} 4$, and $\mathrm{t} 4-\mathrm{t} 5$ time windows. The nonexpert group also showed a significant increase in prediction error from $\mathrm{t} 1$ to $\mathrm{t} 2$. The prediction performance of the experts was superior to that of the nonexperts at all points except $\mathrm{t} 1$ and $\mathrm{t} 5$. The predictions of the expert players were significantly better than chance at all five occlusion conditions on the point-light task, whereas the predictions of the nonexperts at $\mathrm{t} 2$ and $\mathrm{t} 3$ did not differ from guessing levels.

The analysis comparing performance on the point-light task of those participants who had undertaken the film task earlier with that of participants who had not failed to reveal a main effect for previous film task experience, $F(1$, $20)=0.01, p>.05$. Nor was there a significant interaction between that factor and skill level, $F(1,20)=0.02, p>.05$, or between that factor and time of occlusion, $F(4,80)=$ $0.44, p>.05$. Likewise, the analysis comparing the performance on the film task of those participants who had undertaken the point-light task earlier with the performance of those who had not revealed no main effect for previous film task experience, $F(1,20)=0.15, p>.05$; and no significant interaction between that factor and skill level, $F(1,20)=$ $2.21, p>.05$, or between that factor and time of occlusion, $F(4,80)=1.17, p>.05$. Experiencing the film task beforehand afforded participants no advantage or disadvantage in performing the point-light task, and, equally, experiencing 


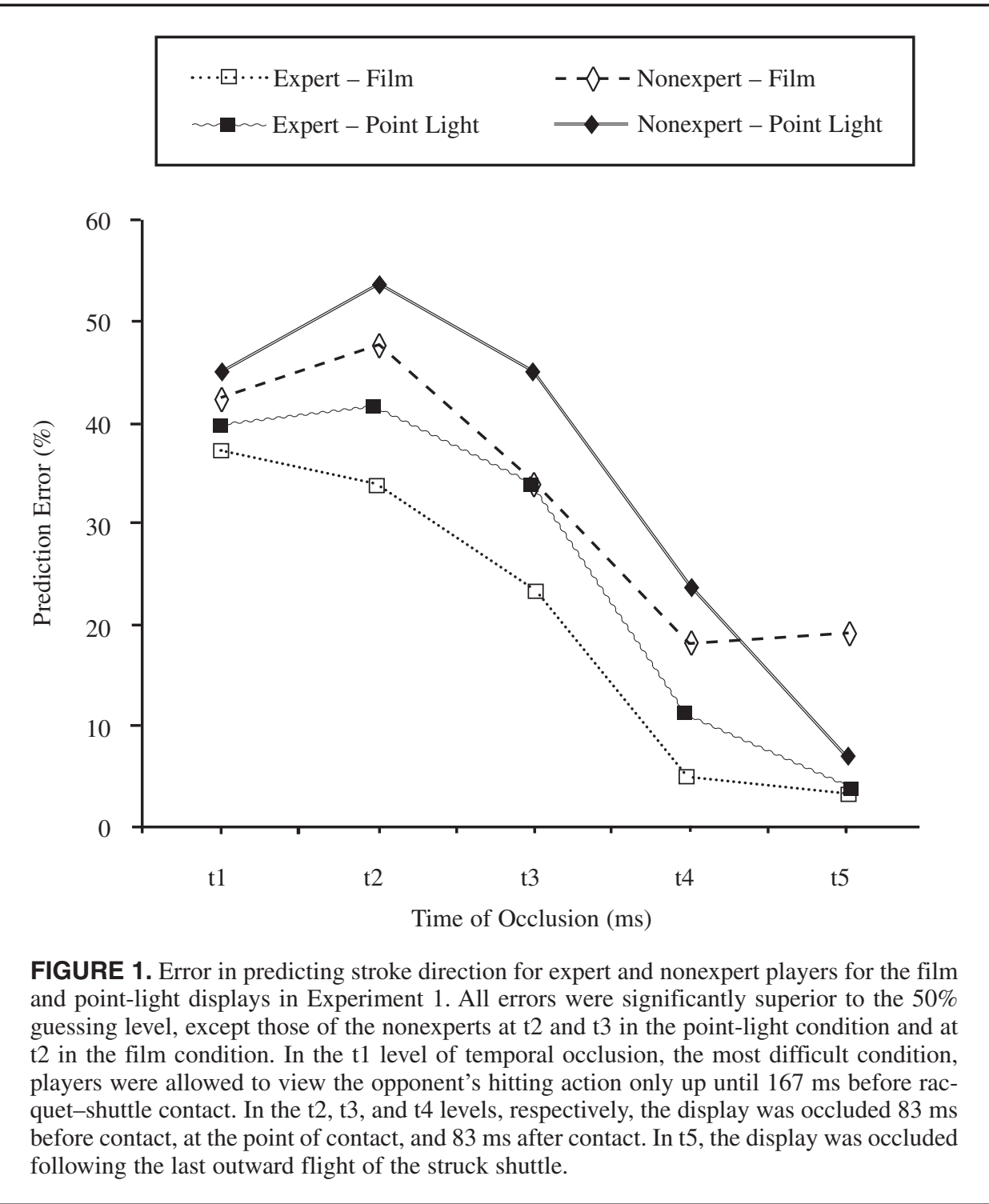

the point-light task beforehand afforded them no advantage or disadvantage in performing the film task.

\section{Discussion}

There was clear evidence that the experts outperformed the nonexperts in prediction of stroke direction, regardless of whether the display was film or point-light, and each skill group demonstrated a comparable pattern of advance information pickup for both the point-light and the film displays. With the exception of the $\mathrm{t} 1$ (for both displays) and the $\mathrm{t} 5$ (for the point-light display) occlusion conditions, the experts produced significantly greater prediction accuracy than did the nonexperts under all occlusion condition and display mode combinations, indicating a superior attunement to the information contained in each of those displays. Like Abernethy et al. (2001) and Ward et al. (2002) in other racquet sports, but unlike Shim et al. (2005), we found that the extent of the expert advantage in advance information pickup did not differ when we used a point-light display or when we used a film display.

Both skill groups, across both tasks, showed a clear ability to use the advance information available in the $\mathrm{t} 2-\mathrm{t} 3$ period and to pick up information before $\mathrm{t} 1$ (prediction accuracy at t1 was better than chance under both display conditions for both groups). The evidence regarding the $\mathrm{t} 1-\mathrm{t} 2$ period was less clear. Across both display conditions, the prediction performance of the experts did not alter significantly from t 1 to $\mathrm{t} 2$, suggesting that no new information pickup was possible for them beyond what was available up to t1. That result differs from the one reported by Abernethy and Russell (1987) and may be attributable to our sample of experts' apparent ability to pick up information before $\mathrm{t} 1$, perhaps making $\mathrm{t} 1-\mathrm{t} 2$ information redundant. In contrast, for the nonexperts, there was evidence of reductions in prediction accuracy from $t 1$ to $\mathrm{t} 2$, suggesting the possibility of information loss through misperception or deception during that period. In the film 
task, the nonexperts' prediction accuracy was superior to guessing level at $\mathrm{t} 1$ but not at $\mathrm{t} 2$. We observed a similar effect in the point-light task: A significant increase in error from $\mathrm{t} 1$ to $\mathrm{t} 2$ resulted in a regression in accuracy from superior to chance at $\mathrm{t} 1$ to no better than guessing level at $\mathrm{t} 2$. Although the performance of the nonexperts was equivalent to that of the experts (and better than chance) at $\mathrm{t} 1$, the prediction accuracy of the nonexperts was significantly inferior to that of the experts on all subsequent occlusion points, with the exception of 15 in the point-light condition. Thus, we needed to determine more precisely the source of the advance information that both experts and nonexperts picked up in the pre-t1 and t2-t3 periods and the cause of the nonexperts' information loss in the $\mathrm{t} 1 \mathrm{-} \mathrm{t} 2$ period.

As we noted previously, the greatest displacement changes of the racquet took place in the $\mathrm{t} 2-\mathrm{t} 3$ period, whereas the greatest displacements of the arm holding the racquet took place during $\mathrm{t} 1-\mathrm{t} 2$ and those of the trunk and lower body took place before $\mathrm{t} 1$. Assuming that the major changes in segment displacement are the most potent sources of information about the movement's kinematics, we suggest on the basis of the results of Experiment 1 that (a) both skill groups are likely able to pick up advance information from the motion of the racquet ( $\mathrm{t} 2-\mathrm{t} 3)$ and from either the lower body or the trunk (pre-t1) and (b) it is the addition of the motion of the arm (in the $\mathrm{t} 1-\mathrm{t} 2$ period) that creates misperceptions among the nonexperts. Information pickup after racquet-shuttle contact in the $\mathrm{t} 3-\mathrm{t} 4$ and $\mathrm{t} 4-\mathrm{t} 5$ periods was, in all probability, attributable to direct information pickup from the flight of the shuttle, and we do not examine that issue further in this article.

\section{EXPERIMENT 2}

Following the general procedures that researchers on gait perception have established (e.g., Cutting, 1978; Cutting, Proffitt, \& Kozlowski, 1978), we sought in Experiments 2 and 3 to isolate more precisely the kinematic information that specifies stroke direction in advance to the skilled observer. In Experiment 2, we sought to determine directly, rather than inferentially, what advance information participants could pick up from the kinematics of selected body segments when we provided vision of point-light displays showing only those segments. We examined information pickup from selected body segments specifically during the period in which those segments underwent their maximal displacements. On the basis of the inferential findings from Experiment 1, we expected that both experts and nonexperts would be able to pick up useful advance information to predict stroke direction from the isolated kinematics of the trunk or lower body (in the period before $\mathrm{t} 1$ ) and from the kinematics of the racquet in isolation (in the period from $\mathrm{t} 2-\mathrm{t} 3$ ). We further expected that the lesser skilled players-but not the experts-would experience an increase in prediction error in the $t 1-t 2$ period when only the kinematics of the arm holding the racquet were visible.

\section{Method}

\section{Procedure}

The procedures and task in Experiment 2 were essentially identical to those in Experiment 1, except that participants viewed incomplete point-light displays showing only the motion of the shuttle and a selected subset of markers (see Figure 2). We presented conditions showing the kinematics of only the racquet (Condition 1), the arm holding the racquet (Condition 2), the upper body (Condition 3 ), or the lower body (Condition 4). Except for the shuttle, we displayed no point-light markers in more than one condition. All conditions again consisted of a total of 160 trials, with occlusions at the same five time periods that we used in the earlier experiment. We counterbalanced the order of presentation of the four conditions across participants and skill groups so that 1 participant was assigned to each of the 24 possible presentation orders and equal numbers of experts and nonexperts experienced each particular condition first.

\section{Data Analysis}

We directed our data analysis in this experiment toward searching for evidence of advance information pickup from each condition within the time period that the displayed feature underwent its maximal change in displacement. Because our interest was only in advance information pickup, we did not analyze data from the $\mathrm{t} 4$ and $\mathrm{t} 5$ occlusion conditions. For Condition 1 (the racquet), we were specifically interested in changes in directional prediction accuracy across the $\mathrm{t} 2-\mathrm{t} 3$ period; for Condition 2 (the arm holding the racquet), we were interested in the $\mathrm{t} 1 \mathrm{-t} 2$ period; and for Conditions 3 (upper body) and 4 (lower body), our interest was in prediction performance up to $\mathrm{t} 1$.

We assessed the evidence for information pickup from the four different displays at select time periods by using planned comparisons in combination with comparison of prediction accuracy levels against the guessing level of $50 \%$. We conducted the planned comparisons (see Table 1) on the directional error data after we had performed an arcsine transformation. For Condition 1, we performed three planned comparisons: one for each of the skill groups between the directional error scores at $\mathrm{t} 2$ and $\mathrm{t} 3$, and one directly comparing the experts with the nonexperts at $\mathrm{t} 3$. We conducted a similar set of comparisons for Condition 2, although our focus in that case was on the $\mathrm{t} 1-\mathrm{t} 2$ period, with the direct skill-group comparison conducted at $\mathrm{t} 2$. For Conditions 3 and 4, we conducted direct comparisons between the two skill groups at $\mathrm{t}$. A conclusion that information pickup had occurred across a particular time window required that two conditions be satisfied: (a) The prediction error at the end of the time window had to be superior to chance, and (b) prediction error had to be significantly reduced from the start of the time window to the end (e.g., for the $\mathrm{t} 1-\mathrm{t} 2$ window, prediction error at $\mathrm{t} 2$ needed to be significantly less than that at $\mathrm{t} 1$ ). The direct skill-group comparisons at the end of the time window enabled us to 


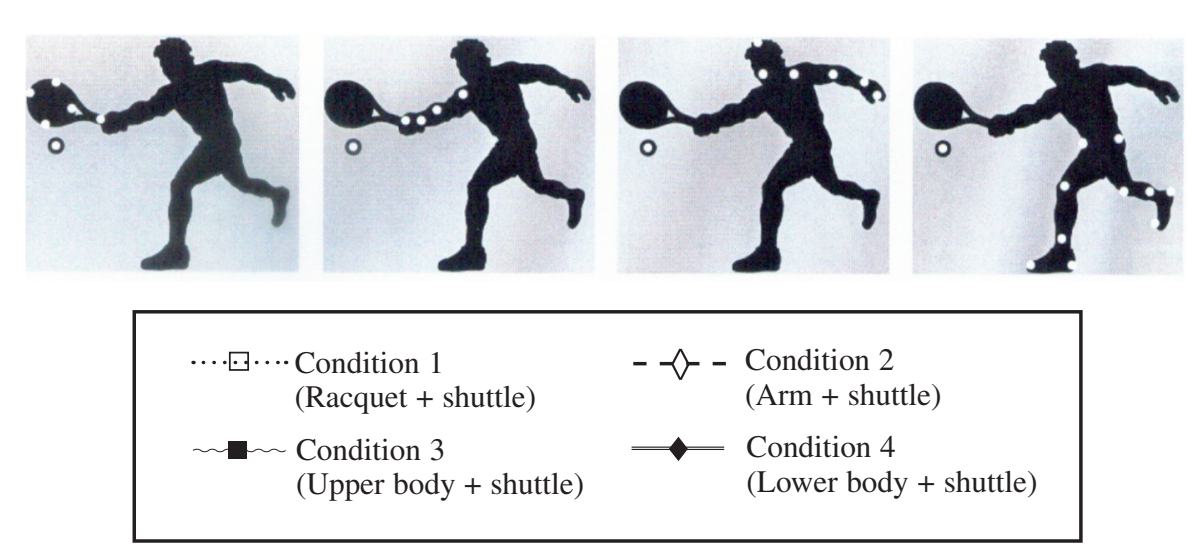

A Experts

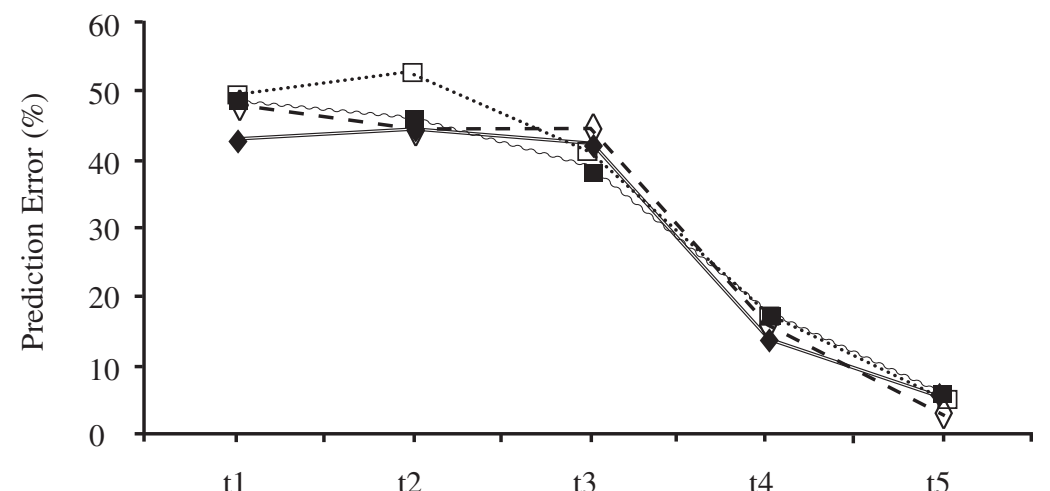

Time of Occlusion (ms)

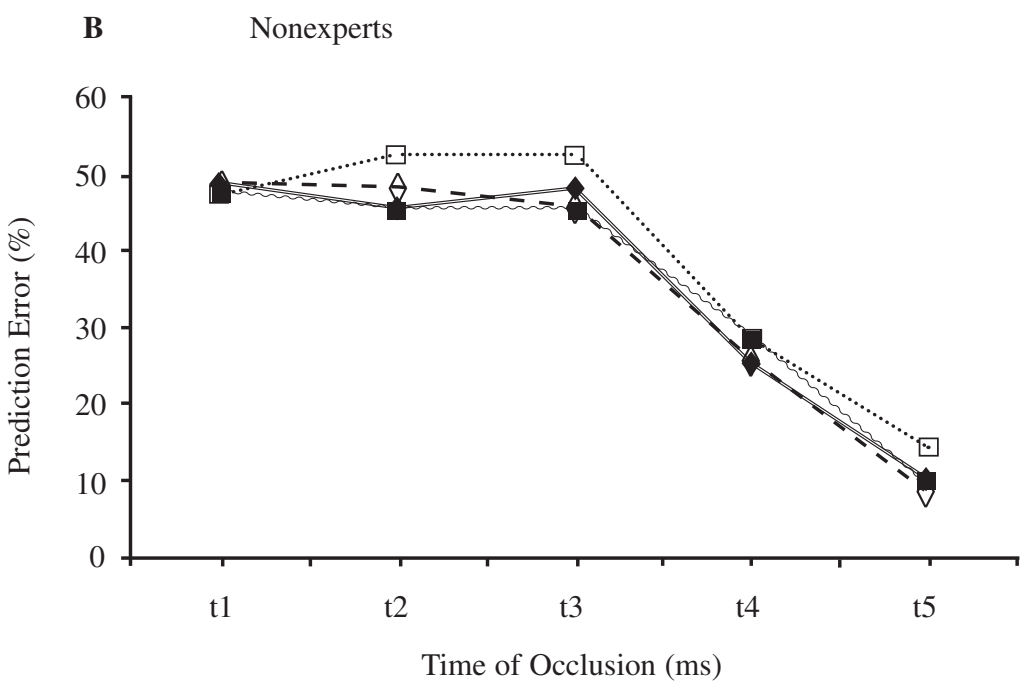

FIGURE 2. Error in predicting stroke direction for (A) expert players and (B) nonexpert players under the four point-light display conditions in Experiment 2. Examples of point-light display Conditions 1-4 are shown from left to right in the top panel. For the experts, all errors for Condition 4 and errors for Condition 1 at t3-t5, Condition 2 at t2-t5, and Condition 3 at t3-t5 were significantly superior to the $50 \%$ guessing level. For the nonexperts, although all conditions had errors significantly superior to the $50 \%$ guessing level at $t 4$ and $t 5$, the only other errors significantly superior to the guessing level were those for Conditions 2 and 3 at $\mathrm{t} 3$ and Condition 4 at $\mathrm{t} 2$. 
determine if any information pickup that occurred across the particular time window, or any of its predecessors, was enough for a significant expert advantage to accrue. The eight planned comparisons we undertook across Experiment 2 were ones that were directly driven by the results of Experiment 1, and they represented only a small subset of the (276) possible comparisons of Skill $\times$ Condition $\times$ Occlusion pairs available. We determined effect sizes for the planned comparisons by using Cohen's $d$ statistic.

\section{Results}

The errors in predicting stroke direction in this experiment are shown separately in Figures $2 \mathrm{~A}$ and B, respectively, for the expert and the nonexpert players. We present the results of the planned comparisons in Table 1.

The collected evidence indicated that the experts-but not the nonexperts-were able to pick up information from the isolated kinematic motion of the racquet presented in Condition 1. Unlike nonexperts, experts significantly reduced their prediction error across the time window in which maximal changes in the racquet kinematics occurred (t2-t3). The experts' pickup of information in this period took their prediction accuracy from no better than chance at $\mathrm{t} 2$ to superior to chance at $\mathrm{t} 3$ and resulted in a significant advantage in prediction accuracy over the nonexperts by $\mathrm{t} 3$. For the nonexperts, prediction accuracy at $\mathrm{t} 3$, like that at $\mathrm{t} 2$, remained no better than chance levels.

There was little evidence that the isolated kinematic motion of the arm holding the racquet (presented in Condi- tion 2) was informative to either skill group. Over the $t 1-t 2$ period in which maximal change in arm position occurred, there was no significant change in prediction performance for either group. The nonexperts' prediction accuracy at both $\mathrm{t} 1$ and $\mathrm{t} 2$ remained at no better than guessing levels, although the experts' prediction at $\mathrm{t} 2$, but not $\mathrm{t} 1$, exceeded chance levels. Any information that experts may have gleaned from the arm motion during the $\mathrm{t} 1-\mathrm{t} 2$ period was of little practical advantage because the prediction accuracy of the expert group was not significantly superior to that of the nonexperts at t2. Similarly, we obtained no evidence to suggest pickup of advance directional information by either skill group from the isolated kinematic motion of the upper body in the period before $\mathrm{t} 1$ (Condition 3). At $\mathrm{t} 1$, when the major upper body motion was completed, neither skill group was able to predict stroke direction at a level better than chance, and the prediction levels of the two groups did not differ.

The major displacements of the lower body (as depicted in Condition 4) occurred before t1. Experts showed an ability to pick up information from the isolated kinematics of the lower body in that period, with prediction accuracies superior to chance achieved by $\mathrm{t} 1$ and sustained thereafter. In contrast the nonexperts' prediction accuracy in Condition 4 was not superior to chance at $\mathrm{t} 1$ and was significantly poorer than that of the experts.

\section{Discussion}

The results of Experiment 2 only partially supported the predictions that we derived from Experiment 1. However,

\begin{tabular}{|c|c|c|c|c|}
\hline Comparison & $d f$ & $t$ & $p$ & $d$ \\
\hline \multicolumn{5}{|c|}{ Condition 1 (racquet + shuttle) } \\
\hline $\mathrm{t} 2$ vs. $\mathrm{t} 3$ for experts & 11 & 2.67 & $.01^{* *}$ & 1.42 \\
\hline $\mathrm{t} 2$ vs. $\mathrm{t} 3$ for nonexperts & 11 & 0.08 & .47 & 0.04 \\
\hline Experts vs.nonexperts@t3 & 22 & 3.78 & $.00^{* *}$ & 1.96 \\
\hline \multicolumn{5}{|c|}{ Condition $2($ arm + shuttle $)$} \\
\hline t1 vs. $\mathrm{t} 2$ for experts & 11 & 1.41 & .09 & 0.67 \\
\hline$t 1$ vs. $t 2$ for nonexperts & 11 & 0.45 & .45 & 0.07 \\
\hline Experts vs. nonexperts @ t2 & 22 & 1.17 & .13 & 0.43 \\
\hline \multicolumn{5}{|c|}{ Condition 3 (upper body + shuttle) } \\
\hline Expertsvsnonexperts@t1 & 22 & 0.06 & .48 & 0.03 \\
\hline \multicolumn{5}{|c|}{ Condition 4 (lower body + shuttle $)$} \\
\hline Experts vs.nonexperts@ @1 & 22 & 2.11 & $.02^{*}$ & 0.95 \\
\hline $\begin{array}{l}\text { Note. One-tailed } t \text { tests are reported } \\
\text { differences between means. Levels } \\
\text { before contact (t2), and point of co } \\
{ }^{*} p<.05{ }^{* *} p<.01 \text {. }\end{array}$ & $\begin{array}{l}11 \text { com } \\
\text { lusion } \\
\text { arge ef }\end{array}$ & $\begin{array}{l}\text { nvolved } \\
\text { ms bef } \\
(d>0.8\end{array}$ & $\begin{array}{l}\text { ediction } \\
\text { t-shuttle } \\
\text { icized. C }\end{array}$ & $\begin{array}{l}\text { ection of } \\
\text { l), } 83 \mathrm{~ms} \\
\text { dition. }\end{array}$ \\
\hline
\end{tabular}


we obtained some skill-related differences in information pickup that were not evident in the earlier experiment. The analysis of the errors that experts made in predicting stroke direction in this experiment indicated that experts pick up and use available information from the isolated kinematics of the racquet (in the period $\mathrm{t} 2-\mathrm{t} 3$ ) and the lower body (in the period before $\mathrm{t} 1$ ). In support of our hypothesis, that result was consistent with the findings of the preceding experiment. We found little evidence of the experts' use of kinematic information from the isolated motion of the arm or the upper body for advance prediction of stroke direction in the period before $\mathrm{t} 1$. In contrast to the experts, and contrary to the expectations from Experiment 1, the nonexperts were unable to make significant information pickup across any adjacent time windows for any of the racquet, arm, upper body, or lower body kinematics when we presented them in isolation from the kinematics of other body segments. Unlike the experts, they appeared to need vision of a relatively full array of kinematic features of the action to pick up anticipatory information.

We obtained no evidence from this experiment to help explain the nonexperts' increase in prediction error during $\mathrm{t} 1-\mathrm{t} 2$ for both the film and point-light displays in Experiment 1. As the segment undergoing major displacement during this period, the arm was the major candidate for generating deception or misperception. However, there was no indication from Condition 2 that the arm was in fact the causal agent. For the nonexperts (and the experts), viewing the isolated arm motion caused neither a significant increase nor a reduction in prediction accuracy, indicating that, at least in isolation, participants made little use of arm motion for advance direction prediction.

The conclusion that experts pick up advance information from racquet and lower body kinematics, whereas lesser skilled players cannot pick up advance information from those or any other isolated kinematic cues, is at odds with the conclusions that Abernethy and Russell (1987) drew from spatial occlusion of precisely the same badminton strokes. There are a number of possible explanations for those differences. First, the experts in the present experiment were more skilled than were those in the study by Abernethy and Russell, and that difference could well account for our experts' ability to use a more advanced source of information (viz., lower body kinematics) than their experts were able to use. Second, there were important differences in method between the two experiments. In the present study, we provided only kinematic information to participants. Abernethy and Russell used film, and nonkinematic information sources such as texture, contour, shape, and color were therefore available, and it is possible that those nonkinematic sources were particularly important for the less skilled players. Furthermore, and perhaps more important, the spatial occlusion method helps determine information loss when a specific feature is masked, whereas the method we used in the present experiment helped us to determine information availability when only the feature of interest was displayed. Therefore, unlike the present method, the spatial occlusion method does not indicate whether a particular feature provides useful information but instead indicates whether the information provided is unique and cannot be gleaned from elsewhere in the display.

\section{EXPERIMENT 3}

Although Experiment 2 provided evidence of expertiserelated differences in attunement to isolated kinematic features, it did not offer insight into the information that each feature can provide when the kinematics of linked segments are also visible, as is the case in the natural setting. If the common-coding hypothesis (e.g., Prinz, 1997) is correct, the availability of vision of linked segments may be important to the facilitation of expert anticipation. In the production of players' overarm throwing patterns, such as those involved in hitting smash shots in badminton, a key aspect of skilled motor performance is the summation and transfer of torques via linked segments - from the larger, more proximal segments of the lower body and trunk to the distal arm and racquet (Elliott, 1995). Following the kinetic chain principle, angular momentum generated in the larger (proximal) body parts is transferred through sequential body segments so that maximal torque and velocity is achieved at the most distal segment, that is, the racquet (Putnam, 1993). As players acquire overarm throwing patterns, degrees of freedom in control are progressively liberated (cf. Bernstein, 1967); segments farther from the end-effector of the hand and arm, such as the trunk and lower body, are progressively recruited so that they can contribute to the action (Gallahue \& Ozmun, 2002). If, as the common-coding view suggests, in their perception and prediction of someone else's overarm hitting action, performers invoke the same neural representations that are responsible for their own production of the comparable action, then the availability of linked segment information may be crucial to both the facilitation of prediction accuracy and the uncovering of further expert advantages.

In Experiment 3, we sought to ascertain whether players could gain additional information for the advance prediction of stroke direction from selected features when the kinematics of adjacent linked segments were present at the same time. We compared prediction accuracies for four different point-light displays in which we varied the number of points that we displayed. With the progressive addition of linked segments, conditions ranged from the simplest one in which only the kinematics of the racquet was visible to a condition in which the kinematics of all segments in the action were displayed. We examined the following three specific questions:

1. Does having contemporaneous vision of the kinematics of the arm holding the racquet and the racquet improve players' prediction performance beyond the level they achieve when only racquet kinematics are visible?

2. Does having contemporaneous vision of the kinematics of the upper body, the arm, and racquet improve players' prediction performance beyond the level they achieve when only arm and racquet kinematics is visible? 
3. Does having contemporaneous vision of the kinematics of the lower body, the upper body, arm, and racquet improve players' prediction performance beyond the level they achieve when only upper body, arm, and racquet kinematics are visible?

Because previous researchers have not addressed those question specifically, the formulation of strong a priori hypotheses was difficult for us. Nevertheless, we predicted on the basis of the common-coding hypothesis that the addition of each of the linked segments would enhance participants' prediction accuracy and might be especially valuable for the more skilled performers.

\section{Method}

\section{Procedure}

We generated two new point-light display conditions for Experiment 3: (a) a display that presented the motion of the joint centers of the arm + racquet + shuttle (Condition 2; see Figure 3) and (b) a display that presented the motion of the upper body + arm + racquet + shuttle (Condition 3; see Figure 3). As in Experiments 1 and 2, we presented 160 trials under each of those display conditions, with the same five levels of temporal occlusion as those used in Experiments 1 and 2. We again required the participants to make predictions of stroke direction. We compared their prediction performances in those two new conditions with their performances in (c) the condition in which we presented only racquet + shuttle information (Condition 1; as per Experiment 2) and (d) the condition in which we presented the lower body + upper body + arm + racquet + shuttle information (Condition 4 , i.e., the point-light display condition from Experiment 1), see Figure 3. We counterbalanced the order of presentation of the two new conditions so that an equal number of participants from each skill group experienced Condition 2 and Condition 3 first.

\section{Data Analysis}

We first transformed the percentage errors in predicting stroke direction by using an arcsine transformation and then conducted planned comparisons between the transformed prediction errors in Conditions 1 and 2, 2 and 3, and 3 and 4 , as detailed in Table 2. We restricted the comparisons to the occlusion time corresponding to the period of maximum spatial change for the segment or the segments that were common to both conditions. Our purpose in conducting the comparisons was to determine whether (a) vision of arm kinematics improved predictions based on racquet kinematics, (b) vision of upper body kinematics improved predictions based on racquet and arm kinematics, and (c) vision of lower body kinematics improved predictions based on racquet, arm, and upper body kinematics, respectively. As before, we also compared levels of prediction accuracy with the guessing level (50\%).

\section{Results}

The errors that the expert and nonexpert players committed in predicting stroke direction are shown in Figure
3. The results of the planned comparisons are presented in Table 2.

We compared prediction performance of the expert and nonexpert groups between Conditions 1 and 2 at $\mathrm{t} 3$ to determine whether contemporaneous vision of the arm helped predictions based on racquet kinematics. Concurrent vision of the arm did not assist experts: Their prediction accuracies at $\mathrm{t} 3$ between the two conditions were indistinguishable. Vision of the arm did assist the nonexperts, however: Their accuracy at $\mathrm{t} 3$ in Condition 2, in which the arm was visible, was significantly superior to that in Condition 1, in which only the racquet was visible. The improvement was such that the expert advantage found at $\mathrm{t} 3$ when only racquet kinematics was visible (see Condition 1, third comparison, in Table 1) was no longer evident when the arm was also visible (third comparison in Table 2).

The comparisons between Conditions 2 and 3 failed to reveal any evidence that participants' contemporaneous vision of the upper body assisted their prediction based on arm and racquet kinematics. For both experts and nonexperts, prediction accuracy at $\mathrm{t} 2$, when major arm displacement had occurred, did not differ between the two conditions. However at $\mathrm{t} 2$ in Condition 3, the experts remained significantly more accurate than the nonexperts and, unlike the nonexperts, produced a prediction accuracy that was superior to guessing levels.

The addition of vision of the lower body (Condition 4) helped both experts' and nonexperts' prediction accuracies as compared with those in Condition 3, in which only upper body, arm, racquet, and shuttle kinematics were visible. For both skill groups, the addition of the lower body information allowed their predictions at $\mathrm{t} 1$ to increase from indistinguishable from guessing to a level significantly superior to that of chance.

\section{Discussion}

The results of Experiment 2 indicated that experts could pick up useful information for the prediction of direction from the local kinematics of the racquet (in t2-t3) and of the lower body (pre-t1). In Experiment 3, we sought to determine whether concurrent vision of the kinematics of more proximal segments of the hitting pattern would enhance participants' information pickup or permit other kinematic features to provide useful advance information. The addition of vision of the arm kinematics to vision of the racquet did not improve the experts' prediction of direction beyond the level they achieved from viewing racquet kinematics alone. Furthermore, the addition of vision of the kinematics of the whole upper body did not improve experts' direction prediction beyond that generated from arm and racquet kinematics. Those findings suggest that if any contribution to experts' advance prediction of stroke direction is possible from arm and upper body kinematics, it is information that is redundant with that from the racquet. In contrast, there was evidence that the addition of lower body kinematics to upper body kinematics did improve participants' prediction 


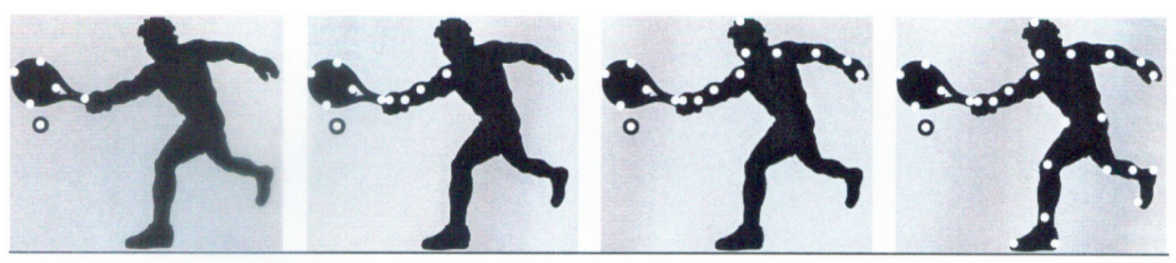

$\cdots \boxminus \cdots$ Condition 1

(Racquet + shuttle)

$\sim$ Condition 3

(Upper body + shuttle) $-\downarrow-$ Condition 2

$($ Arm + shuttle $)$

$\Longrightarrow$ Condition 4

(Lower body + shuttle)

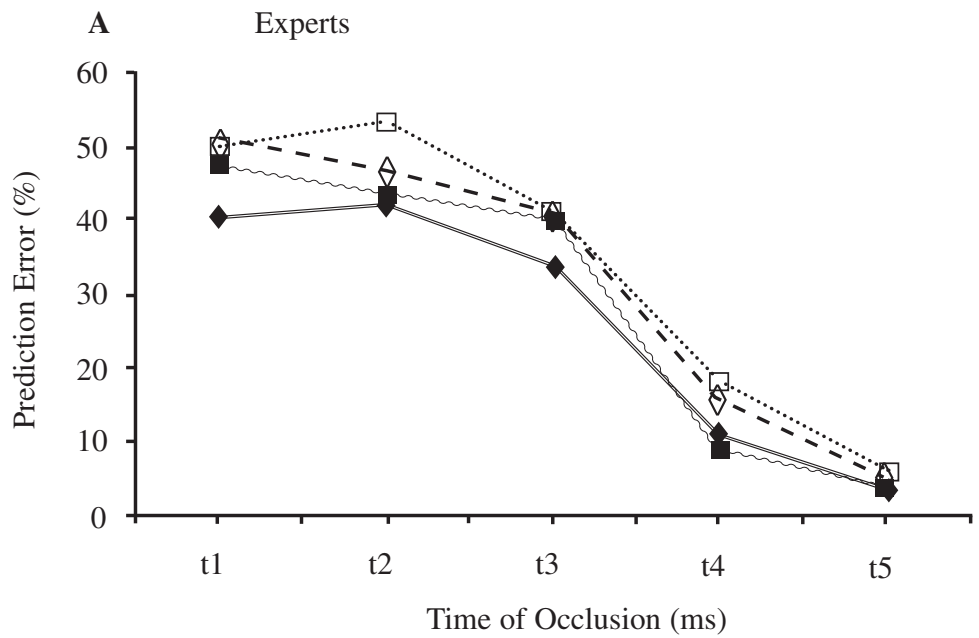

B Nonexperts

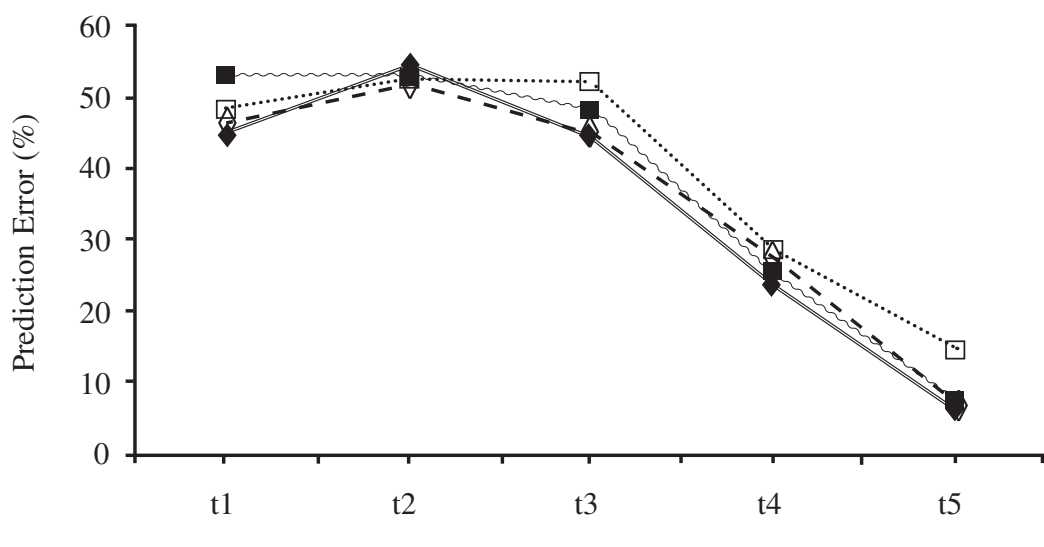

Time of Occlusion (ms)

FIGURE 3. Error in predicting stroke direction for (A) expert players and (B) nonexpert players under the four point-light display conditions in Experiment 3. Examples of point-light display Conditions 1-4 are shown from left to right in the top panel. For the experts, prediction errors superior to guessing levels were evident for occlusion levels t $3-\mathrm{t} 5$ for Condition $1, \mathrm{t} 2-\mathrm{t} 5$ for Conditions 2 and 3, and for all occlusion levels for Condition 4. For the nonexperts, the only precontact occlusion levels under which error was significantly superior to chance were t1 for Condition 4 and 3 for Condition 2. 
TABLE 2. Planned Comparisons for Prediction of Direction in Experiment 3

\begin{tabular}{|c|c|c|c|c|}
\hline Comparison & $d f$ & $t$ & $p$ & $d$ \\
\hline \multicolumn{5}{|c|}{ Conditions 1 and 2} \\
\hline Cond 2 vs. Cond $1 @$ t3 for experts & 11 & 0.00 & .49 & 0.00 \\
\hline Cond 2 vs. Cond $1 @ \mathrm{t} 3$ for nonexperts & 11 & 3.45 & $.00^{* *}$ & 1.52 \\
\hline Experts vs. nonexperts @ t 3 for Cond 2 & 22 & 1.41 & .09 & 0.71 \\
\hline \multicolumn{5}{|c|}{ Conditions 2 and 3} \\
\hline Cond 3 vs. Cond 2 @ 2 for experts & 11 & 0.91 & .19 & 0.32 \\
\hline Cond 3 vs. Cond $2 @ \mathrm{t} 2$ for nonexperts & 11 & 0.29 & .39 & 0.10 \\
\hline Experts vs. Nonexperts @ t 2 for Cond 3 & 22 & 2.44 & $.01^{* *}$ & 1.17 \\
\hline \multicolumn{5}{|c|}{ Conditions 3 and 4} \\
\hline Cond 4 vs. Cond $3 @$ t1 for experts & 11 & 2.09 & $.03^{*}$ & 1.12 \\
\hline Cond4 vs. Cond $3 @ \mathrm{t} 1$ for nonexperts & 11 & 2.37 & $.02^{*}$ & 1.12 \\
\hline
\end{tabular}

Note. One-tailed $t$ tests are reported because all comparisons involved a priori predictions of the direction of differences between means. Conditions (Cond) and occlusion times are described in the text. Large effect sizes $(d>0.80)$ are italicized.

${ }^{*} p<.05 . \quad{ }^{* *} p<.01$.

accuracy compared with their accuracy when only upper body (plus arm and racquet) kinematics was available. That finding suggests that either the lower body motion provides information for experts that is in some way different from that obtainable from the upper body or that the presence of the lower body as a reference facilitates information pickup from the kinematics of the upper body, arm, or racquet. A post hoc comparison revealed no significant difference in prediction accuracies at $\mathrm{t} 1$ for Condition 4 in Experiment 2 (in which only lower body kinematics was present) and for Condition 4 in Experiment 3 (in which lower body kinematics was present along with that of the upper body), $t(11)=$ $1.259, p=.117$ (one-tailed), $d=.457$. That finding suggests that the experts' facilitated prediction noted in Experiment 3 between Conditions 3 and 4 was more likely a consequence of the availability of lower body kinematics, to which (we know from Experiment 2) the experts were attuned, than of the presence of the lower body as a reference point to facilitate early information pickup from the upper body, arm, and racquet.

When we presented the racquet, arm, upper body, and lower body kinematics in isolation in Experiment 2, the nonexperts were unable to use any of those sources to produce better than chance predictions of forthcoming stroke direction. In Experiment 3, we obtained evidence that when vision of the kinematics of the arm and vision of the kinematics of the racquet were available at the same time, significant information pickup was possible. The information pickup was in the $\mathrm{t} 2-\mathrm{t} 3$ period (in which racquet motion was most pronounced) and not in the $\mathrm{t} 1-\mathrm{t} 2$ period (in which arm motion was most pronounced), suggesting that the presence of the arm kinematics facilitated informa- tion pickup from racquet kinematics rather than the reverse. Therefore recreational players' pickup of information from the kinematics of the racquet for the prediction of stroke direction directly depended on the simultaneous presence of the kinematics of the playing-side arm as a kind of reference, whereas experts' did not.

We found no evidence that the nonexperts were able to use the playing-side arm for direction prediction, even with the simultaneous availability of vision of the kinematics of the upper body. There was, however, evidence that lower body kinematics, when present concurrently with that upper body kinematics, enabled the nonexperts to generate a prediction of direction that was superior to the one they generated when only upper body kinematics was visible. The finding that nonexperts were unable to use lower body information when we presented it in isolation in Experiment 2 suggests that the improvement in Experiment 3 most likely resulted from attunement to relational information between the upper body and the lower body. Without a time window before $t 1$, we were unable to ascertain with certainty whether (a) the referential visibility of the lower body improved participants' upper body information pickup or (b) the referential visibility of the upper body allowed participants to pick up lower body information in a way they could not when we presented only upper body or lower body information in isolation.

The common-coding hypothesis supports the proposition that the addition of linked segments (a) enhances prediction accuracy (given that cross-segmental transfer of torques is an integral component of skilled production of the action that we required the participants to predict in this experiment) and (b) is more useful for more skilled 
performers (because of their greater use of cross-segment torque transfers in movement production). Contrary to that proposition, we obtained little evidence in support of the common-coding hypothesis for the experts. In only one case (when the lower body and the upper body, arm, and racquet were visible contemporaneously) was prediction accuracy enhanced by vision of the more proximal linked segment. In that particular case, however, the improvement appeared more attributable to addition of unique information available from the kinematics of the lower body than to the augmentation of information pickup from the more distal segments. In contrast, and counter to expectations from the common-coding hypothesis, vision of linked segments appeared more useful for the nonexperts. For those participants, contemporaneous vision of the kinematics of the arm holding the racquet improved prediction accuracy beyond the level achievable from vision of the racquet kinematics alone, and contemporaneous vision of the kinematics of the lower body improved prediction accuracy more than vision of the upper body, arm, and racquet kinematics alone did.

\section{GENERAL DISCUSSION}

Experiments 1-3 revealed different things about experts' perception and anticipation in badminton. In Experiment 1, we demonstrated that point-light displays convey critical patterns of information pickup, reinforcing the view that an understanding of kinematic information pickup is pivotal to the understanding of skill-related differences in perception. For both skill groups in both the film and point-light tasks, evidence emerged for information pickup in the periods before $\mathrm{t} 1$ and between $\mathrm{t} 2$ and $\mathrm{t} 3$. Furthermore, no improvement in experts' prediction accuracy was evident across the $\mathrm{t} 1-\mathrm{t} 2$ period, whereas nonexperts' prediction accuracy deteriorated across that period. Inference from the major segment displacements within the different time periods suggested that both groups may have been attuned to information from motion of the lower body or trunk (before t1) and the racquet (t2-t 3 ) and that the arm motion (in the $\mathrm{t} 1-\mathrm{t} 2$ period) may have misled the nonexperts. However, we did not directly assess that information pickup from specific segments in Experiment 1. In Experiment 2, we observed skill-related differences in attunement to information arising from the motion of isolated segments. Experts showed an ability to pick up information from the isolated kinematics of the racquet and the lower body, but no such capability was evident for the nonexperts. In Experiment 3, we examined how much the availability of vision of motion from linked segments could facilitate the prediction of stroke direction, and again we obtained evidence of some systematic skill-related differences. Concurrent vision of arm motion improved nonexperts' directional predictions based on racquet kinematics, but not those of experts. For both skill groups, the addition of vision of the lower body kinematics facilitated prediction accuracy based on racquet, arm, and upper body kinematics.

Across key conditions within each of the three experi- ments, the experts were consistently superior to the nonexperts in predictions of stroke direction from point-light displays. An expert advantage in predicting stroke direction was evident at the $\mathrm{t} 1$ occlusion condition when only the lower body kinematics was visible (Condition 4 in Experiment 2); at $\mathrm{t} 2$ when only the upper body, arm, and racquet were present (Condition 3 in Experiment 3) or when all segments were visible (Experiment 1); and at $\mathrm{t} 3$ when all segments were visible (Experiment 1) or when only the racquet was visible (Condition 1 in Experiment 2). In no case was the prediction accuracy of the nonexperts superior to that of the experts.

The defining characteristic of expert performance across the three experiments was the unique ability of the expert players to pick up information from the localized kinematics of the racquet and the lower body. The results of Experiment 3 showed that nonexperts were also able to pick up useful advance information from those two features, but only if linked segment information was also available. For the nonexperts, the addition of vision of motion of the arm segment improved racquet-based predictions, and the addition of vision of the lower body improved the predictions that they could make on the basis of the upper body, arm, and racquet. Comparison of the findings from Experiments 2 and 3 indicates not only that nonexperts may be more dependent on more global sources of information than experts but also that what facilitated improved prediction was not simply vision of more points per se but what specific points were visible. For example, for both skill groups, having more visible points from the upper body did not improve their prediction accuracy (Experiment 3, Condition 2 vs. Condition 3).

Neither skill group showed any evidence of an ability to pick up information from the isolated kinematics of either the arm holding the racquet or the upper body during the periods in which those segments experienced their maximal displacements. However, the arm, when visible concurrently with the racquet, was of use to nonexperts in improving prediction beyond that from the racquet alone, suggesting that the role of the arm is to provide some additional referential or contextual information. The arm was implicated in Experiment 1 as a possible source of nonexperts' loss of $\mathrm{t} 1-\mathrm{t} 2$ information. Our inability to replicate that loss in the later experiments appears to rule out the arm, either in isolation (in Experiment 2) or in tandem with the racquet (in Experiment 3), as the source of that misperception. Neither skill group appeared to pick up useful information for the prediction of stroke direction from the kinematics of the upper body (exclusive of the racquet and playing-side arm). That was true regardless of whether we presented upper body motion in isolation (Experiment 2) or we linked it to the arm and racquet motion (Experiment 3).

The current experiments revealed a capability within world-class badminton players to pick up reliable information for stroke prediction from earlier kinematic events in the opponent's hitting action (i.e., the localized motion 
of the lower body) that previous research has not demonstrated. The experts' active pick up of localized lower body information is consistent with findings from studies of visual search behavior in racquet sports (e.g., Abernethy, 1990) that showed early orientation to that region before racquet fixation. The experts' use of arm information was not apparent in our study, unlike the Abernethy and Russell (1987) study, possibly because our experts' ability to pick up earlier lower body information diminished the importance of pickup of information from the arm. It is noteworthy that our confirmation of the usefulness of lower body kinematics as a source of advance information for experts was possible only because we manipulated the point-light displays in Experiments 2 and 3. The participants' exploitation of those early information sources would not have been apparent if we had used only preexisting methods such as spatial occlusion, unless we had extended those techniques by using embedded temporal occlusion conditions.

Throughout this series of experiments, we gathered evidence demonstrating that experts are superior to lesser skilled performers in predicting stroke direction and that their superiority is related to differences in their attunement to essential kinematic information. Our experiments indicated that experts pick up information from local kinematic features related to the motion of the racquet and lower body that nonexperts are not able to pick up in isolation and that that information pickup is tightly time locked to the segmental involvement of those regions in the biomechanical production of the movement pattern. For example, when players pick up racquet information, it is in the $\mathrm{t} 2-\mathrm{t} 3$ time period when the racquet segment is maximally involved in the hitting action and its major change in displacement is taking place. In the same way, information pickup from the lower body occurs in the time period before $\mathrm{t} 1$ when maximal change in lower body kinematics is taking place. The nonexperts' use of information from the motion of the racquet and lower body is contingent on the availability of other referential and linked features. Overall, consistent with the constraints-attunement hypothesis that Vicente and Wang (1998) developed to explain expertise in memory tasks, experts in racquet sports appear more attuned to the biomechanical constraints of the action they are predicting.

The important consequential challenge to researchers relates to ascertaining how the unique sensitivity of the experts to localized information from the racquet and lower body develops. One proposition that we can advance from the common-coding view (Prinz, 1997) is that the expert players' superior experience and skills in movement production may make possible a concomitant advantage in movement prediction. However, the data collected in this study only partially support that proposition. Although better prediction accuracy occurred overall across many conditions for those participants (the experts) who had greater experience in the production of the type of movement patterns that we asked them to predict, the experts' prediction profiles did not reflect some of the hallmark characteristics of expert movement production. In particular, transfer of torques across linked segments in a proximal-to-distal manner characterizes movement production in racquet sports, yet there was no evidence from Experiment 3 that expertise in prediction was enhanced when linked segments were made visible. To the contrary, nonexperts, rather than experts, appeared to benefit more from the addition of linked segment motion. The defining feature of expertise on the task was not information pickup from segments linked in a manner reminiscent of the linkage in movement production but, rather, unique information pickup from local features (the racquet and lower body) that contain kinematic information predictive of future shot direction. More detailed analyses of the kinematics of the hitting action in badminton are now necessary for researchers to identify more precisely which kinematic characteristics of the racquet and lower body motion provide the advance information to which only the experts are attuned.

\section{ACKNOWLEDGMENTS}

An Australian Research Council grant to Bruce Abernethy and a University Putra study grant to Khairi Zawi partially supported the studies reported in this article. The present research formed part of Khairi Zawi's doctoral studies at the University of Queensland. The authors acknowledge the statistical advice of Ester Cerin.

\section{NOTE}

1. Participants also made a prediction about stroke depth, but we have reported that information in detail elsewhere (Abernethy \& Zawi, 2007).

\section{Biographical Notes}

Bruce Abernethy is Director of the Institute of Human Performance at University of Hong Kong and researches movement expertise and the perception and production of patterns of human movement.

Khairi Zawi teaches skill acquisition at the University Putra, Malaysia.

\section{REFERENCES}

Abernethy, B. (1990). Expertise, visual search and information pick-up in squash. Perception, 19, 63-77.

Abernethy, B., Gill, D., Parks, S. L., \& Packer, S. T. (2001). Expertise and the perception of kinematic and situational probability information. Perception, 30, 233-252.

Abernethy, B., \& Russell, D. G. (1987). Expert-novice differences in an applied selective attention task. Journal of Sport Psychology, 9, 326-345.

Abernethy, B., \& Zawi, K. (2007). Expertise and attunement to kinematic constraints. Manuscript submitted for publication.

Bartlett, F. C. (1947, June 14). The measurement of human skill. British Medical Journal, 835-838, 877-880.

Bernstein, N. (1967). The coordination and regulation of movement. London: Pergamon Press.

Brownlow, S., Dixon, A. R., Egbert, C. A., \& Radcliffe, R. D. (1997). Perception of movement and dancer characteristics from point-light displays of dance. Psychological Record, 47, 411-421.

Buccino, G., Binkofski, F., \& Riggio, L. (2004). The mirror neuron system and action recognition. Brain and Language, 89, 370-376. 
Buccino, G., \& Riggio, L. (2006). The role of the mirror neuron system in motor learning. Kinesiology, 38, 5-15.

Calvo-Merino, B., Glaser, D. E., Grèzes, J., Passingham, R. E., \& Haggard, P. (2005). Action observation and acquired motor skills: An fMRI study with expert dancers. Cerebral Cortex, 15, 1243-1249.

Cutting, J. E. (1978). Generation of male and female synthetic walkers through manipulation of a biomechanical invariant. Perception, 7, 393-405.

Cutting, J. E., \& Kozlowski, L. T. (1977). Recognizing friends by their walk: Gait perception without familiarity cues. Bulletin of the Psychonomic Society, 9, 353-356.

Cutting, J. E., Proffitt, D. R., \& Kozlowski, L. T. (1978). A biomechanical invariant for gait perception. Journal of Experimental Psychology: Human Perception and Performance, 4, 357-372.

Elliott, B. C. (1995). The biomechanics of tennis stroke production. In T. Reilly (Ed.), Science and racket sports (pp. 89-97). London: E. \& F. N. Spon.

Enberg, M.-L. (1968). Assessing perception of object directionality in tennis (Doctoral dissertation, Purdue University, 1968). Dissertation Abstracts International, 29, 806-A.

Gallahue, D. L., \& Ozmun, J. C. (2002). Understanding motor development: Infants, children, adolescents, adults (5th ed.). Boston: McGraw-Hill.

Gowitzke, B. A., \& Waddell, D. B. (1979). Qualitative analysis of the badminton forehand smash as performed by international players. Proceedings of a National Symposium on Racquet Sports (pp. 10-25). Chicago, IL: University of Illinois.

Gray, S., Watts, S., Debicki, D., \& Hore, J. (2006). Comparison of the kinematics in skilled and unskilled arms of the same recreational baseball players. Journal of Sports Sciences, 24, 1183-1194.

Hecht, H., Vogt, S., \& Prinz, W. (2001). Motor learning enhances perceptual judgement: A case for action-perception transfer. Psychological Research, 65, 3-14.

Hommel, B., Musseler, J., Aschersleben, G., \& Prinz, W. (2001). The theory of event coding (TEC): A framework for percep- tion and action planning. Behavioral and Brain Sciences, 24, 849-878.

Johansson, G. (1973). Visual perception of biological motion and a model for its analysis. Perception and Psychophysics, 14, 201-211.

Kourtzi, Z., \& Shiffar, M. (1999). Dynamic representations of human body movement. Perception, 28, 49-62.

Kreighbaum, E., \& Barthels, K. M. (1996). Biomechanics: A qualitative approach for studying human movement. Needham Heights, MA: Allyn \& Bacon.

Prinz, W. (1997). Perception and action planning. European Journal of Cognitive Psychology, 9, 129-154.

Putnam, C. (1993). Sequential motions of body segments in striking and throwing skills: Descriptions and explanations. Journal of Biomechanics, 26, 125-135.

Sakurai, S., \& Ohtsuki, T. (2000). Muscle activity and accuracy of performance of the smash stroke in badminton with reference to skill and practice. Journal of Sports Sciences, 18, 901-914.

Salthouse, T. A. (1991). Expertise as the circumvention of human processing limitations. In K. A. Ericsson \& J. Smith (Eds.), Toward a general theory of expertise: Prospects and limits (pp. 286-300). Cambridge, England: Cambridge University Press.

Shim, J., Carlton, L. G., Chow, J. W., \& Chae, W-S. (2005). The use of anticipatory visual cues by highly skilled tennis players. Journal of Motor Behavior, 37, 164-175.

Vicente, K. J., \& Wang, J. H. (1998). An ecological theory of expertise effects in memory recall. Psychological Review, 105, $33-57$.

Ward, P., Williams, A. M., \& Bennett, S. J. (2002). Visual search and biological motion perception in tennis. Research Quarterly for Exercise and Sport, 73, 107-112.

Submitted March 17, 2006

Revised September 23, 2006

Second revision February 14, 2007 


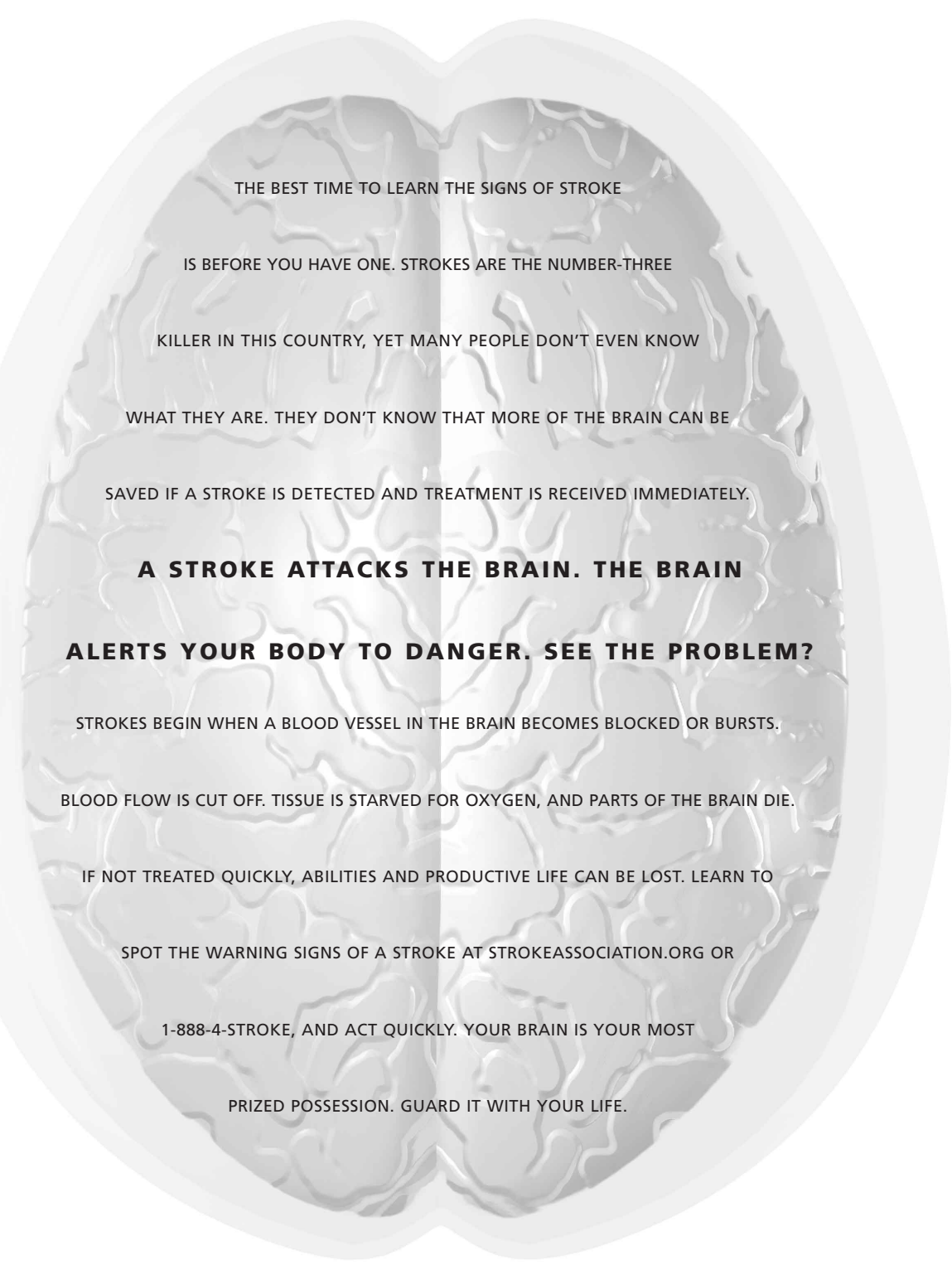

WITH A STROKE, TIME LOST IS BRAIN LOST.

If you suddenly have or see any of these symptoms, call 9-1-1 immediately: Numbness or weakness of the face, arm or leg, especially on one side of the body - Confusion, trouble speaking or understanding - Difficulty seeing in one or both eyes - Trouble walking, dizziness, loss of balance or coordination - Severe headache with no known cause

Learn more at StrokeAssociation.org or 1-888-4-STROKE. Association 
Copyright of Journal of Motor Behavior is the property of Heldref Publications and its content may not be copied or emailed to multiple sites or posted to a listserv without the copyright holder's express written permission. However, users may print, download, or email articles for individual use. 